Reprod. Nutr. Dévelop., 1986, 26 (2 B), 649-658.

\title{
Lipogenèse des tissus adipeux de chevreaux sevrés à 4,6 et 8 semaines
}

\author{
P. BAS, Annie ROUZEAU, P. MORAND-FEHR
}

Station de Nutrition et Alimentation (I.N.R.A.), Institut National Agronomique Paris-Grignon, 16. rue Claude-Bernard, 75231 Paris Cedex 05.

Summary. Lipogenesis in adipose tissues of kids weaned at 4, 6 and 8 weeks.

Adipose deposits and their lipogenic enzymes were studied on 27 young Alpine male kids. They were weaned either at 28, 42 or 56 days of age and for each age at weaning, 3 animals were slaughtered unweaned $(P 0)$, or $14(P+14)$ or $56(P+56)$ days after weaning. They received milk replacer ad libitum from day 5 after birth to weaning and concentrate, hay and water from a week before weaning to slaughter.

The adipose tissues were very developed in the abdomen in $\mathrm{P} 0$ and $\mathrm{P}+56$ animals. Subcutaneous tissues were scarce. All adipose tissues in the $P+14$ were lighter. The older the animals, the greater was the reduction in adipose tissue weight between $P 0$ and $P+14$. But this loss was proportionally higher in younger animals. In all $P+14$ animals the perirenal tissues showed higher loss.

Lipoprotein lipase (LPL) activity was high in abdominal adipose tissues of $\mathrm{P} 0$ and $P+56$ kids, particularly in the omental tissue, but was very low in sternal adipose tissue. LPL activity had a low value 14 days after weaning but its specific activity roughly reached the unweaned LPL value 56 days after weaning.

By contrast, acetyl CoA carboxylase (ACX) activity was very low in omental adipose tissue but existed even in unweaned kids. Fifty-six days after weaning, this activity was high in 28-day weaned kids. The activity of both glucose-6-phosphate dehydrogenase (G6PDH) and malic enzyme (EM) was high in unweaned kids, particularly at 42 days. Surprisingly, EM activity was higher than G6PDH activity in kids weaned for 56 days. Thus, in ad libitum concentrate-fed kids, the ATP-citrate lyase EM pathway seemed to be active and important for lipogenesis.

\section{Introduction.}

Au sevrage, le jeune chevreau passe d'une alimentation liquide à un régime à base de fourrages et d'aliments concentrés nettement moins riche en lipides et de faible densité énergétique. En outre, ce jeune ruminant est en déficit énergétique en raison des quantités ingérées limitées dans les jours qui suivent la suppression du lait. Ce déficit est d'autant plus marqué que le chevreau est sevré jeune ou à un faible poids (Fehr, 1972 ; Fehr et Sauvant, 1976). Au sevrage, le métabolisme du ruminant doit s'adapter à utiliser les acides gras volatils, issus des fermenta- 
tions du rumen, comme source d'énergie et à produire le glucose qui n'est plus absorbé au niveau de l'intestin.

L'évolution des activités enzymatiques liées aux métabolismes lipidique et glucidique ont été étudiées dans le foie d'agneaux et de veaux en liaison avec l'âge (Ballard et Oliver, 1965 ; Bartley, Freedland et Black, 1966) ou le sevrage (Howarth, Baldwin et Ronning, 1968). En revanche, dans le tissu adipeux, l'adaptation du métabolisme lipidique en relation avec la croissance et le développement des fonctions digestives du rumen n'a été que partiellement abordée chez les jeunes ruminants (Howarth, Baldwin et Ronning, 1968 ; Bas, Rouzeau et MorandFehr, 1985).

II est couramment admis que le cycle citrate/malate/pyruvate est négligeable chez les ruminants, ce qui est en rapport avec la faible incorporation du glucose dans les lipides de ces animaux (Hanson et Ballard, 1967 ; Ingle, Bauman et Garrigus, 1972). Or même lorsque les activités des enzymes de ce cycle ont pu être mesurées, leurs valeurs ont été extrêmement faibles et peu adaptatives avec des régimes riches en glucides (Baldwin et al., 1966; Young, Thorp et de Lumen, 1969).

Dans la présente étude, nous avons examiné les répercussions de l'âge au sevrage sur la croissance des tissus adipeux et sur l'évolution de l'activité de quelques enzymes intervenant dans la lipogenèse du tissu adipeux.

\section{Matériel et méthodes.}

Vingt-sept chevreaux mâles de race Alpine sont répartis en 3 lots, de 9 animaux, équilibrés chacun suivant le poids à la naissance, le gain de poids des 12 premiers jours, la taille de la portée et l'origine parentale. Dès le 5 e jour, ils reçoivent du lait reconstitué avec un aliment d'allaitement à la concentration de $15 \%(P / P)$, distribué chaud, à volonté une fois par jour. De la semaine précédant le sevrage jusqu'à l'abattage, les animaux reçoivent du foin de luzerne, de l'aliment concentré sous forme granulée et de l'eau.

Les animaux des trois lots sont sevrés respectivement à 28,42 ou 56 jours (S28, S42, S56). Dans chaque lot, 3 animaux sont abattus au sevrage ou à 14 ou 56 jours après le sevrage ( $P 0 ; P+14, P+56$ ).

A l'abattage, les tissus adipeux abdominaux (omental, périrénal et mésentérique) et le tissu sous-cutané sternal sont prélevés et pesés. Des parties aliquotes sont conservées à $-50^{\circ} \mathrm{C}$.

L'activité de la lipoprotéine lipase (LPL) : EC 3.1.1.34 est mesurée dans tous ces tissus selon la méthode de Chilliard, Dorléans et Fehr (1977). Les activités de l'acétyl CoA carboxylase (ACX) : EC 6.4.1.2, de l'enzyme malique (EM, NADP + malate déhydrogénase) : EC 1.1.1.40 et de la glucose-6-phosphate déhydrogénase (G6PDH) : EC 1.1.1.49 sont estimées dans le tissu omental respectivement par les méthodes de Pascal et al. (1977), de Hsu et Lardy (1969) et de Ficht, Hill et Chaikoff (1959). 


\section{Résultats.}

Poids des tissus adipeux (tabl. 1).

Chez tous les chevreaux étudiés, les lipides s'accumulent tout particulièrement dans les tissus adipeux de la cavité abdominale alors qu'au contraire les lipides sous-cutanés restent peu développés comme en témoigne la faible masse du tissu sternal comparée à celles des tissus adipeux abdominaux.

TABLEAU 1

Evolution du poids de quatre tissus adipeux du chevreau autour du sevrage.

\begin{tabular}{|c|c|c|c|c|c|c|c|c|c|}
\hline \multicolumn{2}{|c|}{ Tissus adipeux } & \multicolumn{2}{|c|}{ Omental } & \multicolumn{2}{|c|}{ Périrénal } & \multicolumn{2}{|c|}{ Mésentérique } & \multicolumn{2}{|c|}{ Sternal } \\
\hline $\begin{array}{l}\text { Lots } \\
S\left(^{*}\right)\end{array}$ & $P\left({ }^{* *}\right)$ & $\bar{x}(g)\left(^{* * *}\right)$ & C.V. $\left({ }^{* * *}\right)$ & $\bar{x}$ & C.V. & $\bar{x}$ & C.V. & $\bar{x}$ & C.V. \\
\hline 28 & $\begin{array}{r}0 \\
14 \\
56\end{array}$ & $\begin{array}{l}84,0 \\
20,3 \\
85,3\end{array}$ & $\begin{array}{l}(31,7) \\
(64,2) \\
(85,3)\end{array}$ & $\begin{array}{r}83,7 \\
6,7 \\
54,7\end{array}$ & $\begin{array}{l}(19,6) \\
(16,4) \\
(50,5)\end{array}$ & $\begin{array}{r}75,3 \\
44,3 \\
164,3\end{array}$ & $\begin{array}{l}(23,4) \\
(15,0) \\
(26,8)\end{array}$ & $\begin{array}{r}6,3 \\
1,7 \\
13,7\end{array}$ & $\begin{array}{l}(24,3) \\
(69,5) \\
(66,0)\end{array}$ \\
\hline 42 & $\begin{array}{r}0 \\
14 \\
56\end{array}$ & $\begin{array}{l}189,3 \\
108,7 \\
262,0\end{array}$ & $\begin{array}{l}(20,8) \\
(24,2) \\
(40,9)\end{array}$ & $\begin{array}{r}164,7 \\
67,7 \\
140,0\end{array}$ & $\begin{array}{l}(53,0) \\
(33,5) \\
(53,1)\end{array}$ & $\begin{array}{l}119,3 \\
115,0 \\
271,0\end{array}$ & $\begin{array}{r}(37,9) \\
(9,0) \\
(18,6)\end{array}$ & $\begin{array}{r}13,7 \\
7,7 \\
30,0\end{array}$ & $\begin{array}{l}(21,0) \\
(61,3) \\
(35,3)\end{array}$ \\
\hline 56 & $\begin{array}{r}0 \\
14 \\
56\end{array}$ & $\begin{array}{l}269,3 \\
191,0 \\
323,0\end{array}$ & $\begin{array}{r}(18,6) \\
(26,9) \\
(9,7)\end{array}$ & $\begin{array}{l}312,3 \\
116,3 \\
126,0\end{array}$ & $\begin{array}{l}(31,4) \\
(37,7) \\
(21,2)\end{array}$ & $\begin{array}{l}162,3 \\
169,0 \\
307,0\end{array}$ & $\begin{array}{l}(13,7) \\
(12,0) \\
(13,3)\end{array}$ & $\begin{array}{r}16,0 \\
9,7 \\
30,7\end{array}$ & $\begin{array}{r}(16,6) \\
(26,0) \\
(9,4)\end{array}$ \\
\hline
\end{tabular}

$\left({ }^{*}\right) \mathrm{S}=$ Age au sevrage (jours); $\left({ }^{*}\right) \mathrm{P}=$ Durée de la période post-sevrage (jours) ; $\left({ }^{* *}\right) X=$ moyenne $(n=3) ;\left(n^{* * *}\right)$ C.V. = Coefficient de variation.

Avant le sevrage, les tissus abdominaux se développent rapidement de façon linéaire entre 28 et 56 jours. Le poids total de ces tissus (omental, périrénal et mésentérique) est de 253, 496 et $778 \mathrm{~g}$ respectivement à 28,42 et 56 jours, soit $2,7,4,2$ et $4,9 \%$ du poids vif vide.

Quatorze jours après le sevrage $(P+14)$, tous les tissus adipeux étudiés ont fortement régressé, excepté le tissu mésentérique dans le cas des animaux sevrés à 42 et 56 jours. La baisse de poids de ces tissus est plus importante chez les animaux sevrés à 42 ou 56 jours que chez ceux sevrés à 28 jours. Mais, rapportée au poids de ces tissus au sevrage ( $P$ ), cette baisse est beaucoup plus marquée chez les animaux du lot S28. Dans les 3 lots, le tissu périrénal est le tissu adipeux dont le poids baisse le plus au cours des 2 semaines qui suivent le sevrage $(P+14)$.

Cinquante-six jours après le sevrage $(P+56)$, les tissus adipeux omental et mésentérique et le tissu sous-cutané sternal ont un poids supérieur à celui observé au sevrage $(P 0)$. Cette augmentation de poids est plus nette dans les tissus mésentérique et sternal ( $P$ : NS, $P<0,001$ et $P<0,001$ ) respectivement pour les tissus omental, mésentérique et sternal. A l'inverse, le poids du tissu adipeux 
périrénal est significativement plus faible $(P<0,05) 56$ jours après le sevrage que chez les animaux non sevrés.

\section{Activités spécifiques des enzymes lipogéniques}

a) Lipoprotéine lipase (tabl. 2). - L'activité spécifique (par g de tissu frais) de la LPL, qui catalyse le prélèvement des acides gras des lipoprotéines sériques de faible densité (VLDL et chylomicrons), subit de grandes variations après le sevrage.

\section{TABLEAU 2}

Evolution de l'activité spécifique de la lipoprotéine lipase dans les tissus adipeux du chevreau autour du sevrage.

\begin{tabular}{|c|c|c|c|c|c|c|c|c|c|}
\hline \multicolumn{2}{|c|}{ Tissus adipeux } & \multicolumn{2}{|c|}{ Omental } & \multicolumn{2}{|c|}{ Périrénal } & \multicolumn{2}{|c|}{ Mésentérique } & \multicolumn{2}{|c|}{ Sternal } \\
\hline $\begin{array}{l}\text { Lots } \\
S\left({ }^{*}\right)\end{array}$ & $P\left({ }^{* *}\right)$ & $\bar{x}$ & C.V. & $\bar{x}$ & C.V. & $\bar{x}$ & C.V. & $\bar{x}$ & C.V. \\
\hline 28 & $\begin{array}{r}0 \\
14 \\
56\end{array}$ & $\begin{array}{l}460 \\
110 \\
593\end{array}$ & $\begin{array}{l}(74,7) \\
(59,5) \\
(7,4)\end{array}$ & $\begin{array}{l}383 \\
136 \\
597\end{array}$ & $\begin{array}{l}(66,5) \\
(65,9) \\
(24,6)\end{array}$ & $\begin{array}{r}224 \\
17 \\
164\end{array}$ & $\begin{array}{l}(27,1) \\
(77,1) \\
(18,8)\end{array}$ & $\begin{array}{l}92 \\
22 \\
82\end{array}$ & $\begin{array}{l}(96,0) \\
(61,1) \\
(36,7)\end{array}$ \\
\hline 42 & $\begin{array}{r}0 \\
14 \\
56\end{array}$ & $\begin{array}{l}596 \\
124 \\
367\end{array}$ & $\begin{array}{l}(21,3) \\
(116) \\
(31,4)\end{array}$ & $\begin{array}{l}396 \\
151 \\
365\end{array}$ & $\begin{array}{l}(28,9) \\
(124) \\
(34,0)\end{array}$ & $\begin{array}{r}262 \\
82 \\
172\end{array}$ & $\begin{array}{l}(35,8) \\
(64,4) \\
(22,4)\end{array}$ & $\begin{array}{r}173 \\
42 \\
43\end{array}$ & $\begin{array}{l}(30,6) \\
(51,5) \\
(88,2)\end{array}$ \\
\hline 56 & $\begin{array}{r}0 \\
14 \\
56\end{array}$ & $\begin{array}{r}408 \\
76 \\
503\end{array}$ & $\begin{array}{l}(77,4) \\
(84,0) \\
(38,0)\end{array}$ & $\begin{array}{r}219 \\
51 \\
424\end{array}$ & $\begin{array}{l}(77,0) \\
(59,1) \\
(20,1)\end{array}$ & $\begin{array}{l}220 \\
104 \\
290\end{array}$ & $\begin{array}{r}(89,7) \\
(80,4) \\
(1,0)\end{array}$ & $\begin{array}{r}116 \\
34 \\
64\end{array}$ & $\begin{array}{l}(65,3) \\
(31,1) \\
(26,7)\end{array}$ \\
\hline
\end{tabular}

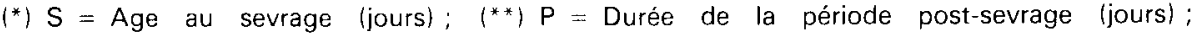
$\mathrm{LPL}$ : Lipoprotéine Lipase $(\mathrm{nm} / \mathrm{mn} / \mathrm{g})$.

Avant le sevrage, cette activité diffère significativement selon les tissus. Elle est la plus élevée dans le tissu omental. Elle est plus faible dans les tissus périrénal et mésentérique que dans le tissu omental (respectivement le tiers et la moitié environ). Dans le tissu sous-cutané sternal, l'activité spécifique de la LPL est nettement plus faible que dans les tissus internes $(20$ à $30 \%$ de celle du tissu omental).

Au cours des 2 semaines qui suivent le sevrage l'activité spécifique de la LPL baisse fortement. A P +14 , l'activité LPL est d'environ 20,30 et $25 \%$ de celle des animaux non sevrés respectivement pour les tissus omental, périrénal et sternal. Dans le tissu mésentérique, l'activité LPL est d'autant plus abaissée 14 jours après le sevrage, que les animaux ont été sevrés plus jeunes. De $P+14$ à $P+56$, l'activité spécifique de la LPL dans les tissus internes croît à nouveau pour atteindre des valeurs voisines de celles des animaux non sevrés. Dans le tissu sternal, l'activité de la LPL ne croît que faiblement de $P+14$ à $P+56$ sans jamais atteindre celle des animaux $P 0$ et spécialement ceux sevrés à 42 ou 56 jours. 
Huit semaines après le sevrage, la LPL des tissus adipeux abdominaux atteint des niveaux proches ou légèrement supérieurs à ceux des animaux $P$ 0. Toutefois, dans le tissu sous-cutané sternal, l'activité spécifique de la LPL est restée significativement plus faible $(P<0,05)$ qu'avant le sevrage.

b) Enzymes de la lipogenèse de novo (tabl. 3). - Les activités de l'ACX, de la G6PDH et de l'EM du tissu omental présentent des variations assez comparables à celles de la LPL. Ces activités subissent une chute dans les 14 jours qui suivent le sevrage. Cette baisse est plus marquée pour la G6PDH dont l'activité est plus élevée avant le sevrage $(P<0,01)$. Elle est significative au seuil de $5 \%$ pour l'EM mais n'est pas significative pour l'ACX. La reprise d'activité mesurée au $56^{e}$ jour après le sevrage est relativement plus nette lorsque le sevrage est pratiqué à 28 jours plutôt qu'à 42 ou 56 jours pour la G6PDH et l'EM, mais il semble que ce soit le contraire pour l'ACX.

TABLEAU 3

Evolution de l'activité spécifique de trois enzymes de la lipogenèse de novo $(A C X G 6 P D H, E M)$ dans le tissu omental du chevreau autour du sevrage.

\begin{tabular}{|c|c|c|c|c|c|c|c|}
\hline \multirow{2}{*}{$\begin{array}{l}\text { Enzymes } \\
\text { Lots } \\
\quad\left(S^{*}\right)\end{array}$} & \multirow[b]{2}{*}{$P(* *)$} & \multicolumn{2}{|c|}{$A C X$} & \multicolumn{2}{|c|}{ G6PDH } & \multicolumn{2}{|c|}{ EM } \\
\hline & & $\bar{x}$ & C.V. & $\bar{x}$ & C.V. & $\bar{x}$ & C.V. \\
\hline 28 & $\begin{array}{r}0 \\
14 \\
56\end{array}$ & $\begin{array}{l}4,1 \\
2,2 \\
4,6\end{array}$ & $\begin{array}{l}(23,5) \\
(126) \\
(72,3)\end{array}$ & $\begin{array}{l}1,1 \\
0,2 \\
0,7\end{array}$ & $\begin{array}{l}(22,2) \\
(106) \\
(28,7)\end{array}$ & $\begin{array}{l}0,4 \\
0,2 \\
0,9\end{array}$ & $\begin{array}{l}(28,7) \\
(91,6) \\
(18,5)\end{array}$ \\
\hline 42 & $\begin{array}{r}0 \\
14 \\
56\end{array}$ & $\begin{array}{l}3,2 \\
2,6 \\
6,2\end{array}$ & $\begin{array}{l}(65,6) \\
(37,5) \\
(40,4)\end{array}$ & $\begin{array}{l}2,8 \\
0,3 \\
0,5\end{array}$ & $\begin{array}{l}(38,6) \\
(35,5) \\
(29,1)\end{array}$ & $\begin{array}{l}2,2 \\
0,9 \\
0,8\end{array}$ & $\begin{array}{l}(7,7) \\
(41,7) \\
(21,1)\end{array}$ \\
\hline 56 & $\begin{array}{r}0 \\
14 \\
56\end{array}$ & $\begin{array}{r}3,3 \\
2,9 \\
13,6\end{array}$ & $\begin{array}{l}(144) \\
(103) \\
(43,3)\end{array}$ & $\begin{array}{l}1,1 \\
0,3 \\
0,6\end{array}$ & $\begin{array}{l}(94,8) \\
(33,5) \\
(20,2)\end{array}$ & $\begin{array}{l}1,6 \\
0,6 \\
0,9\end{array}$ & $\begin{array}{l}(51,0) \\
(38,0) \\
(53,4)\end{array}$ \\
\hline
\end{tabular}

$\left(^{*}\right) S=$ Age au sevrage (jours); $\left(^{* *}\right) \mathrm{P}=$ Durée de la période post-sevrage (jours).

$\mathrm{ACX}=$ Acétyl CoA Carboxylase $(\mathrm{nm} / \mathrm{mn} / \mathrm{g}$ de tissu frais); G6PDH = Glucose 6 phosphate déhydrogénase $(\mu \mathrm{m} / \mathrm{mn} / \mathrm{g}) ; E M=$ Enzyme malique $(\mu \mathrm{m} / \mathrm{mn} / \mathrm{g})$.

L'activité de l'ACX du tissu omental est peu influencée par l'âge des animaux au sevrage. En revanche, elle est la plus élevée $(P<0,10)$ chez les animaux les plus âgés qui ont aussi été sevrés le plus tardivement.

Les activités de la G6PDH et de l'EM sont les plus élevées chez les animaux non sevrés abattus à 42 jours. Chez les animaux non sevrés, la G6PDH semble plus active que l'EM à 28 et à 42 jours mais pas à 56 jours. Après le sevrage, I'EM est généralement plus active que la G6PDH.

Les activités de I'EM et de la G6PDH semblent être très liées $(r=+0,77$; $P 0,001 ; n=27)$. Mais si chez les animaux non sevrés elles ne sont corrélées ni avec I'ACX ni avec la $L P L$, en revanche, 56 jours après le sevrage, leurs niveaux d'activités sont corrélés avec la $L P L(r: E M / L P L=+0,82, P<0,01$ et 
$r: G 6 P D H / L P L=0,60, P<0,10, n=9$ cf. fig. 1 et 2) mais indépendantes de I'ACX ( $r$ : EM/ACX et G6PDH/ACX $<0,2$ NS).

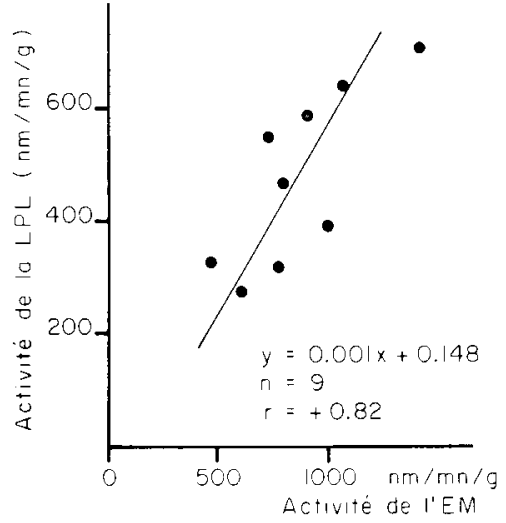

FIG. 1. - Relations entre les activités $\angle P L$ et EM dans le tissu adipeux omental de chevreaux sevrés depuis 8 semaines.

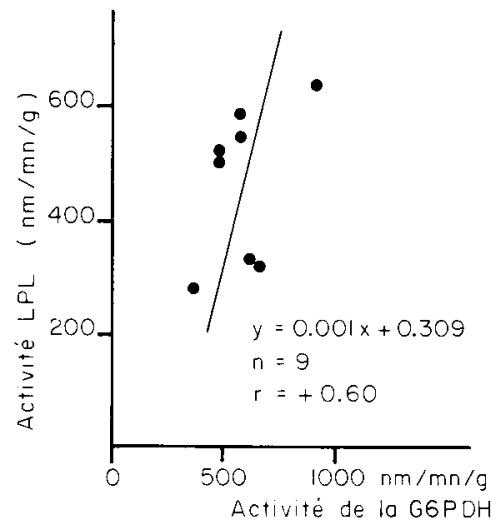

FIG. 2. - Relations entre les activités $L P L$ et G6PDH dans le tissu adipeux omental de chevreaux sevrés depuis 8 semaines.

\section{Discussion.}

Cette étude confirme que les chevreaux de race laitière stockent en grande partie leurs réserves énergétiques dans la cavité abdominale et qu'au contraire, la masse des tissus adipeux sous-cutanés reste négligeable y compris chez des animaux abattus à des poids élevés (entre 25 et $30 \mathrm{~kg}$ : sous lot S56-P56).

Pendant la période de restriction alimentaire consécutive au sevrage, tous les tissus étudiés perdent du poids mais le tissu adipeux qui a toujours le plus régressé est le tissu périrénal comme cela avait déjà été suggéré par les résultats de Bas et al. (1985) sur jeunes caprins, de Jacob et al. (1973) sur ovins et de Seebeck (1973) sur bovins.

Les chevreaux sevrés le plus précocément, c'est-à-dire à 4 semaines, mobilisent la plus grande partie des lipides stockés dans les tissus adipeux pendant la période d'allaitement, au cours des 2 semaines qui suivent le sevrage.

Pendant la période de croissance comprise entre 14 et 56 jours après le sevrage, les dépôts de lipides restent relativement limités, comparés à ceux des chevreaux de même âge non sevrés (Bas et al., 1985). Une plus faible ingestion d'énergie et un taux moindre de lipides dans la ration des animaux récemment sevrés semblent responsables du développement plus réduit des tissus adipeux, chez les animaux sevrés.

Le chevreau n'accumule pas les lipides dans les mêmes tissus avant et après le sevrage. Après le sevrage, les lipides sont surtout déposés dans les tissus adipeux omental et mésentérique, et beaucoup moins dans le tissu périnal.

La LPL présente une activité très élevée dans les tissus adipeux internes des chevreaux alors que dans le tissu sous-cutané sternal son activité est relativement faible. La faible activité de la LPL chez les animaux abattus 2 semaines après le 
sevrage confirme la sensibilité de cette enzyme au niveau nutritionnel de l'animal comme cela a été montré précédemment sur chevreaux soit après $24 \mathrm{~h}$ de jeûne (Morand-Fehr et al., 1985), soit après un sevrage brutal (Bas et al., 1985).

Si avant sevrage la forte activité LPL dans les tissus adipeux internes peut être reliée au taux de lipides de la ration, en revanche, 8 semaines après le sevrage, les valeurs élevées de la LPL sont surprenantes chez des animaux dont la ration est pauvre en lipides, même si les triglycérides sanguins contiennent une part non négligeable d'acides gras synthétisés dans le rumen (Sutton, Storry et Brumby, 1979) et, sous certaines conditions, dans le foie (Ballard, Filsell et Jarrett, 1972). Aussi le fait que, d'une part, l'activité LPL soit élevée chez tous les animaux 8 semaines après le sevrage, et que, d'autre part, son activité spécifique soit aussi élevée dans les tissus omental et périrénal dont les vitesses de croissance sont différentes, renforce la suggestion de Bas et al. (1985), selon laquelle l'activité de la LPL mesurée in vitro ne serait pas liée à la quantité d'acides gras prélevés par les tissus adipeux. Si cette enzyme semble avoir un rôle important pour le développement des réserves lipidiques chez le chevreau comme l'indiquent Haugeback, Hedrik et Asplund (1974) et Vézinhet, Nougues et Teyssier (1983), en revanche, elle ne doit pas être un facteur limitant les prélèvements des acides gras des triglycérides sanguins chez le chevreau en croissance.

Dans le tissu omental, qui s'est le plus développé après le sevrage, l'ACX contrairement à la LPL, est restée très peu active. Cette disproportion entre les activités des enzymes LPL et $A C X$ est du même ordre que celle rapportée par Vézinhet et al. (1983) chez l'agneau en croissance. Avant sevrage, le taux élevé de lipides de la ration a probablement inhibé l'activité de l'ACX comme cela avait été montré par Vernon (1976) chez des ovins recevant un régime riche en lipides. Par contre, les valeurs de I'ACX restent faibles après le sevrage, même chez les animaux les plus âgés. Le faible niveau de ces valeurs est à relier au fait que I'ACX est généralement considérée comme une enzyme limitante pour la synthèse des acides gras chez les ruminants (Ingle et al., 1973). Mais les conditions opératoires généralement employées (inhibition par les ions chlorure du milieu) réduisent l'activité de l'ACX (Allred et Roehrig, 1980 ; Bas, Houlier et Rouzeau, résultats non publiés).

Si l'activité de l'ACX semble très faible dans le tissu adipeux de chevreau, en revanche celle des enzymes liées à la production de NADPH est très élevée. L'aliment lacté apportant une quantité importante de lipides à l'organisme (environ $100 \mathrm{~g}$ /jour), les activités de ces enzymes pourraient ne pas refléter la lipogenèse de novo.

Chez les animaux non sevrés, les activités spécifiques de la G6PDH et de I'EM présentent un pic vers 6 semaines comme chez le rat (Gandemer, Pascal et Durand, 1979) et chez le porc (Anderson et Kauffman, 1973). Cependant, l'amplitude du pic d'activité de ces enzymes est plus modeste que chez le rat et le porc. L'âge de l'animal semble donc être un des principaux responsables de l'accroissement de l'activité de ces enzymes ainsi que l'avaient suggéré Taylor, Bailey et Bartley (1967). La faible activité de l'EM, à l'âge de 3 semaines, peut paraître surprenante puisque d'après les résultats de Hanson et Ballard (1968), l'EM est 10 fois plus active dans le foie de fœtus de bœuf que dans celui du rat. 
Ces enzymes étant fortement réduites après le sevrage, la forte disponibilité en glucose chez les animaux non sevrés est probablement responsable de leurs activités élevées avant le sevrage.

Deux semaines après le sevrage, la baisse d'activité de la G6PDH et de l'EM semble être due à la restriction alimentaire consécutive au sevrage lorsque l'animal s'est adapté à l'alimentation solide. Elle est du même ordre que celle provoquée par le jeûne (Ingle et al., 1973 ; Pothoven et Beitz, 1975).

Huit semaines après le sevrage, le niveau élevé de ces enzymes peut résulter de la production importante de précurseurs glucogéniques comme le propionate provenant de l'amidon du régime lorsque l'aliment concentré (à $36 \%$ de céréales) est fourni à volonté. L'induction de ces enzymes à NADPH par le glucose ou ses précurseurs a déjà été préalablement démontrée chez les ruminants par Ballard, Filsell et Jarret (1972) ; Prior et Scott (1980) et Smith et al. (1984). Un taux élevé de propionate pourrait d'autre part expliquer les liaisons étroites observées entre les activités LPL et celles des déhydrogénases à NADP par un effet activateur de ce précurseur glucogénique sur ces enzymes. Le fait que l'EM soit plus active que la G6PDH chez les animaux sevrés depuis 8 semaines, nous conduit à penser que les modalités d'induction de ces 2 enzymes peuvent être différentes. La G6PDH pourrait être plus liée à la disponibilité en glucose et I'EM à celle des précurseurs glucogéniques. Par ailleurs, l'induction de l'EM n'est probablement maximale qu'après plusieurs mois (Smith et al., 1984) et pourrait être exacerbée chez les animaux en croissance ayant consommé peu d'aliments grossiers par rapport aux aliments concentrés. Ces facteurs conjugués expliqueraient que I'EM soit plus active que la G6PDH comme l'ont observé Martin et Wilson (1974).

Ainsi, la voie ATP citrate lyase-enzyme malique peut devenir fonctionnelle comme l'ont montré Whitehurst et al. (1978), Smith et Prior (1981) et Smith et al. (1984). Les enzymes de cette voie semblent particulièrement actifs chez le jeune ruminant lorsque le régime post-sevrage reste toujours riche en amidon. Dans ces conditions, il est permis de penser que lorsque les précurseurs glucogéniques sont abondants ceux-ci fournissent non seulement des équivalents réducteurs pour la synthèse des acides gras mais aussi une part non négligeable des maillons acéty! de leur squelette carboné.

11 Réunion du groupe Développement I.N.R.A., Montpellier, 22-24 mai 1985.

\section{Références}

ALLRED J., ROEHRIG K. L., 1980. Inhibition of rat liver acetyl CoA by chloride. J. Lipid Res., 21, 488-491.

ANDERSON D. B., KAUFFMAN R. G., 1973. Cellular and enzymatic changes in porcine adipose tissue during growth. J. Lipid Res., 14, 160-168.

BALDWIN R. L., RONNING M., RADANOVICS C., PLANGE G., 1966. Effect of carbohydrate and fat intakes. J. Nutr., 90, 47-55.

BALLARD F. J., FILSELL O. H., JARRETT I. G., 1972. Effect of carbohydrate availability on lipogenesis in sheep. Biochem. J., 226, 193-200.

BALLARD F., OLIVER J. T., 1965. Carbohydrate metabolism in liver from fetal and neonatal sheep. Biochem. J., 95, 191-200. 
BARTLEY J. C., FREEDLAND R. A., BLACK A. L., 1966. Effect of aging and glucose loading on the activities of glucose-6-phosphatase an phosphorylase of livers of cows and calves. Amer. J. vet. Res., 27, 1243-1248.

BAS P., ROUZEAU A., MORAND-FEHR P., 1985. Poids et métabolisme des réserves lipidiques au cours de la croissance du chevreau. Reprod. Nutr. Dévelop., 25, 275-285.

CHILLIARD Y., DORLÉANS M., FEHR P. M., 1977. Mise en évidence d'une activité lipoprotéine lipasique dans le tissu adipeux de chèvre : Comparaison de trois méthodes d'extraction. Ann. Biol. anim. Bioch. Biophys., 17, 107-122.

FEHR P. M., 1972. Répercussions du poids au sevrage sur les performances de croissance des chevrettes. //e Congr. mond. Alimentation animale Madrid, octobre 1972, vol. V. p. 363-373.

FEHR P. M., SAUVANT D., 1976. Production de chevreaux lourds. I. Influence de l'âge et du mode de sevrage sur les performances des chevreaux abattus à 26,5-29 kg. Ann. Zootech., 25, 243257.

FICHT W. N., HILL R., CHAIKOFF J. L., 1959. The effect of glucose feeding on glycolytic enzyme activities of the normal rat liver. J. biol. Chem., 234, 1048-1051.

GANDEMER G., PASCAL G., DURAND G., 1979. Developmental changes in lipogenic enzyme activities in liver and adipose tissue of post-weaning rats. Effect of sex and castration. Ann. Biol, anim. Bioch. Biophys., 19, 573-581.

HAUGEBAK C. D., HEDRICK H. B., ASPLUND J. M., 1974. Relationship between extramuscular adipose tissue lipoprotein lipase activity and intramuscular lipid deposition in fattening lambs. J. anim. Sci, 39, 1026-1031.

HANSON R. W., BALLARD F. J., 1967. The relative significance of acetate and glucose precursors for lipid synthesis in liver and adipose tissue from ruminants. Biochem. J., 105, 529-536.

HANSON R. W., BALLARD F. J., 1968. The metabolic fate of the products of citrate cleavage. Adenosine triphosphate-citrate lyase and nicotinamide-adenine dinucleotide phosphate-linked malate dehydrogenase in foetal and adult liver from ruminants and non-ruminants. Biochem. J., 108, 705-713.

HOWARTH R. E., BALDWIN R. L., RONNING M., 1968. Enzyme activities in liver, muscle and adipose tissue of calves and steers. J. Dairy Sci., 51, 1270-1274.

HSU R. Y., LARDY H. A., 1969. Malic enzyme. In LOWENSTEIN J. M., Methods in enzymology, 17, 230-235, Acad. Press, New-York, London.

INGLE D. L., BAUMAN D. E., GARRIGUS U. S., 1972. Lipogenesis in the ruminant: in vitro study of tissue sites, carbon source and reducing equivalent generation for fatty acid synthesis. J. Nutr., 102, 609-616.

INGLE D. L., BAUMAN D. E., MELLENBERGER R. W., JOHNSON D. E., 1973. Lipogenesis in the ruminant : Effect of fasting and refeeding on fatty acid synthesis and enzymatic activity of sheep adipose tissue. J. Nutr., 103, 1479-1488.

JACOB J. A., FIELD R. A., BOTKIN M. P., RILEY H. L., 1973. Effect of dietary stress on lamb carcass composition and quality. $J$. anim. Sci, 36, 507-510.

MARTIN R. J., WILSON L. L., 1974. Comparison of tissue enzyme levels and carcass characteristics in Hereford and Holstein steers. J. anim. Sci, 39, 865-870.

MORAND-FEHR P., BAS P., ROUZEAU A., HERVIEU J., 1985. Development and characteristics of adipose deposits in male kids during growth from birth to weaning. Anim. Prod., 41, 349357.

PASCAL G., DURAND G., MACAIRE J. P., PÉNOT R., 1977. Evolution de l'activité de l'acetyl-CoAcarboxylase et de la liprotéine lipase dans le foie et les tissus adipeux du rat mâle au cours du développement après sevrage. Influence du taux de lipides de la ration. Ann. Biol. anim. Bioch. Biophys., 17, 827-849.

POTHOVEN M. A., BEITZ D. C., 1975. Changes in fatty acid synthesis and lipogenic enzymes in adipose tissue from fasted and fasted-refed steers. J. Nutr., 105, 1055-1061.

PRIOR R. L., SCOTT R. A., 1980. Effects of intravenous infusions of glucose, lactate, propionate or acetate on the induction of lipogenesis in bovine adipose tissue. J. Nutr., 110, 2011-2019.

SEEBECK R. M., 1973. The effect of body-weight loss on the composition of Brahman cross and Africander cross steers. II. Dissected components of the dressed carcass. J. agric. Sci, $\mathbf{8 0}$, 411-423.

SMITH S. B., PRIOR R. L., 1981. Evidence for a functionnal ATP-citrate lyase/NADP malate 
dehydrogenase pathway in bovine adipose tissue enzyme and metabolite levels. Arch. Biochem. Biophys., 211, 192-201.

SMITH S. B., PRIOR R. L., FERREL C. L., MERSMANN H. J., 1984. Inter-relationships among diets, age, fat deposition and lipid metabolism in growing steers. J. Nutr., 114, 153-162.

SUTTON J. D., STORRY J. E., BRUMBY P. E., 1979. Rumen microbial synthesis of long-chain fatty acids. Proc. Nutr. Soc., 38, 4A.

TAYLOR C. B., BAILEY E., BARTLEY W., 1967. Changes in hepatic lipogenesis during development of the rat. Biochem. J., 105, 717-722.

VERNON R. G., 1976. Effect of dietary fats on ovine adipose tissue metabolism. Lipids, 11, 662-669.

VERNON R. G., 1980. Lipid metabolism in the adipose tissue of ruminant animals. Progr. Lipid. Res., 19, 23-106.

VÉZINHET A., NOUGUĖS J., TEYSSIER J., 1983. Influence d'un mode d'élevage sur la mise en réserve des graisses par le tissu adipeux chez les ovins. Reprod. Nutr. Dévelop., 23, 837-846.

WHITEHIRST G. B., BEITZ D. C., POTHOVEN M. A., ELLISON W. R., CRUMP M. H., 1978. Lactate as a precursor of fatty acids in bovine adipose tissue. J. Nutr., 108, 1806-1811.

YOUNG W., THORP S. L., de LUMEN H. Z., 1969. Activity of selected gluconeogenic and lipogenic enzymes in bovine rumen mucosa, liver and adipose tissue, Biochem. J., 114, 8388. 